\title{
Neue Funktion der Austrittsberichte durch TARPSY
}

\section{Jürg Unger-Köppel}

Dr. med. , Mitglied des FMH-Zentralvorstandes, Departementsverantwortlicher Stationäre Versorgung und Tarife

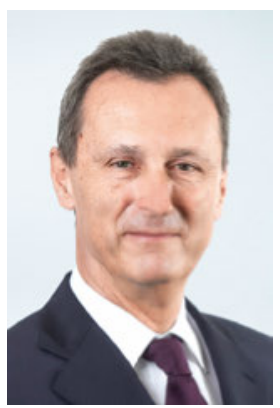

Ab dem 1.Januar 2018 soll in der stationären Erwachsenenpsychiatrie neu nach TARPSY abgerechnet werden. Somit erfolgt auch in der Psychiatrie die Umstellung von den klassischen Tagespauschalen auf ein neues System mit diagnosespezifischen degressiven Tagessätzen. Dieses bringt die Leistung und deren Abgeltung in einen näheren Bezug zueinander, wie es das Krankenversicherungsgesetz KVG fordert. Vor kurzem stimmten die Tarifpartner dem Tarifstrukturvertrag $\mathrm{zu}$, der unter anderem regelt, wie damit umgegangen wird, falls eine Institution nach Umstellung des Systems sehr viel mehr oder sehr viel weniger einnehmen sollte. Damit ist auch die letzte Voraussetzung erfüllt, dass der Bundesrat den Tarif zeitgerecht für die Einführung per 1. Januar 2018 bewilligen kann.

Die Diagnose und einzelne Symptome werden einen unmittelbaren Einfluss auf die Vergütung haben.

Swiss Mental Health Care (SMHC), die Vereinigung der institutionellen Psychiatrien in der Schweiz, führte Ende Juni 2017 eine sehr gut besuchte Tagung durch, die sich der Vorbereitung der Organisationen auf die Einführung des TARPSY widmete. Es zeigte sich, dass die Ärzteschaft und die Administration wichtige Aufgaben zu lösen haben bis zum Start anfangs 2018. Die Verantwortlichen für die Verhandlungen mit den Krankenkassen können nicht davon ausgehen, dass die heutige Tagespauschale in den Basispreis überführt wird. Sie müssen sehr viel komplexere Berechnungen vorlegen, um ein gutes Verhandlungsergebnis für ihre Institution zu erreichen.

Auch die Ärzteschaft sollte sich gut vorbereiten: Bis heute wurden wahrscheinlich an den wenigsten Orten ausserhalb des Teams Kaderarzt - Assistenzarzt im Alltag die einzelnen Diagnosen der Patienten vertieft überprüft, solange eine klinische Besserung des $\mathrm{Pa}$ tienten eintrat. Das wird sich nun ändern, indem die Diagnose und einzelne Symptome einen unmittelbaren Einfluss auf die Vergütung haben werden: Je nach gestellten klinischen Diagnosen wird die psychiatrische Klinik mehr oder weniger Geld einnehmen. Dieser Bezug zwischen Diagnose und Einnahmen bestand in der Periode der klassischen Tagespauschalen nicht die Pauschale war für alle Diagnosen die gleiche. Diese Systemänderung wurde in der Somatik schon 2012 mit der Einführung der DRG vollzogen.

Wegen dem allerorts herrschenden Einnahmedruck könnten die Diagnostizierenden dazu verführt werden, vermehrt jene Diagnosen zu stellen, die höhere Erträge generieren. Deshalb wird zur Kontrolle die sogenannte Kodierrevision eingeführt: unabhängige Externe überprüfen bei zufälligen Stichproben auf Grund derselben Unterlagen, die den Kodierenden zur Verfügung standen, ob die Kodierung plausibel ist. Der Bericht über die Kodierqualität wird anschliessend der Institution sowie den Kassen und Kantonen zugänglich gemacht. Das erklärte Ziel der Verantwortlichen der Psychiatrie ist, dass die Kodierung und die Kodierrevision nur auf Grund des Austrittsberichtes erfolgen soll und nicht auf Grund der ganzen Krankengeschichte mit intimen Details, die für die Diagnosestellung nicht entscheidend sind. Damit bekommt der Austrittsbericht in der Psychiatrie eine zweite Funktion: Neben den wesentlichen Informationen für die

\section{Der Austrittsbericht enthält nicht nur Infor-} mationen zur Nachbehandlung, sondern stellt neu auch die Basis für die Kodierung dar.

Nachbehandelnden muss er neu auch alle Informationen beinhalten, die die Basis für die richtige Kodierung gewährleisten. Es empfiehlt sich, dass die Ärzteschaft unter Leitung der Chefärzte die verbleibende Zeit bis Ende Jahr nutzt, um den Prozess zur Verfassung der Austrittsberichte unter die Lupe zu nehmen und sicherzustellen, dass die Berichte den neuen Anforderungen genügen. $\mathrm{Ob}$ die Nachbehandelnden den ausführlichen Bericht, der die Basis für die Kodierung und deren Revision bildet, erhalten, oder nur einen Auszug, wird die Praxis zeigen. 